\title{
On-Orbit Servicing Mission Operations at GSOC
}

\author{
S. Eberle ${ }^{1}$, A. Ohndorf ${ }^{2}$ and R. Faller ${ }^{3}$ \\ German Space Operations Center \\ DLR Oberpfaffenhofen \\ 82230 Wessling, Germany
}

\begin{abstract}
On-Orbit Servicing (OOS) is not only an option for the repair and the upgrade of space assets that suffer from technical failures but might also be a promising business case, e.g. extending the lifetime of geostationary communication satellites. Another example of robotic service operations would be the servicing of low-earth orbit satellites. Currently, this expertise is being developed at GSOC in order to operate the Orbit Life Extension Vehicle (OLEV) and the German Orbital Servicing Mission (DEOS). The multinational commercial mission OLEV will achieve this task by docking a smaller servicer spacecraft to the apogee engine nozzle of a regular geostationary communication satellite and performing the station keeping task for a number of additional years in service and a de-orbiting at the end. The mission features a novel space-application of interesting technologies like high level autonomy and robotics. Contrary to the other mission phases, a different network architecture must be used because of the challenging telepresence (short latency) requirement. This requirement holds also true for the technology demonstration mission DEOS, as its robotic docking, berthing and de-berthing operations of the two spacecrafts are to be carried out by video-based telepresence. Due to the different orbit heights of both missions, the resulting requirements on the network differ significantly. Whereas in DEOS both spacecrafts (servicer and client) are controlled by one team at GSOC, OLEV is characterized by operating the satellites with very close coordination between GSOC and the client control center. This paper presents the special features and resulting distinctive challenges of these missions and how GSOC copes with them during the mission preparation, i.e. how they are reflected in the ground system design as well as the flight operations of the respective phases.
\end{abstract}

$\begin{array}{ll} & \\ \text { DEOS } & =\text { DEutsche Orbitale Servicing Mission } \\ \text { FOT } & =\text { Flight Operations Team } \\ \text { GEO } & =\text { GEostationary Orbit } \\ \text { GSOC } & =\text { German Space Operation Center } \\ \text { HST } & =\text { Hubble Space Telescope } \\ \text { LEO } & =\text { Low Earth Orbit } \\ \text { LEOP } & =\text { Launch and Early Orbit Phase } \\ \text { OLEV } & =\text { Orbital Life Extension Vehicle } \\ \text { OOS } & =\text { On-Orbit Servicing } \\ \text { PCS } & =\text { Payload Control Subsystem } \\ \text { RvD } & =\text { Rendezvous \& Docking } \\ \text { SSC } & =\text { Swedish Space Cooperation } \\ \text { SST } & =\text { Satellite Support Team } \\ \text { TC } & =\text { TeleCommand } \\ \text { TM } & =\text { TeleMetry }\end{array}$

\footnotetext{
${ }^{1}$ Project Manager, German Space Operations Center, DLR Oberpfaffenhofen, 82230 Wessling, Germany, sabrina.eberle@dlr.de

${ }^{2}$ Project Engineer,German Space Operations Center, DLR Oberpfaffenhofen, 82230Wessling,Germany, andreas.ohndorf@dlr.de

${ }^{3}$ Project Manager, German Space Operations Center, DLR Oberpfaffenhofen, 82230 Wessling, Germany, ralf.faller@dlr.de 


\section{Introduction}

Today, satellites in Low Earth Orbit (LEO) gather data of the Earth's surface and its atmosphere to provide researchers and decision makers with information. The orbit height of such satellites is typically between $200 \mathrm{~km}$, where the upper atmosphere blends into space, and 500-700 km. Space objects whose orbit lies in or crosses the LEO band, are still exposed to the Earth's atmosphere, which has a very low, but still non-negligible, density at this height. Depending on the initial perigee distance, it takes from months to hundreds of years for an object to slow down, and thus lower its orbit height, until it finally, depending on the objects size and mass, burns up due to the increasing air friction when re-entering the atmosphere. As this is desired for nowadays existing man-made debris in orbits around Earth, it can evolve into a serious problem if larger structures or heavier satellites re-enter the atmosphere as they do not incinerate completely. If the spacecraft is then uncontrollable, also the impact area on Earth cannot be influenced in order to minimize potential hazard to inhabited areas. Nevertheless, according to international agreements, every space-faring nation is responsible for any object it launches into space over its whole lifetime in space. Thus it becomes necessary to develop techniques to enable a controlled re-entry for so-called noncooperative objects.

Another growing field of interest is the ability to access and service high-value space assets, e.g. expensive communication or scientific satellites, or telescopes like the Hubble Space Telescope (HST). The concept of an Experimental Servicing Satellite (ESS), a first example of what is now called On-Orbit Servicing (OOS), was presented already in $1998^{1}$. This idea was an outcome of the failed German TV-Sat 1 mission of 1987, in which the downlink antenna could not be deployed. In general, OOS means, first, to extent the lifetime of satellites in danger of becoming uncontrollable, e.g. due to propellant shortage, or to replace defect items, or both. Second, OOS offers a chance to upgrade satellite hardware and thus to enhance its capabilities, something that is done already regularly with onboard software. Of course, this needs to be taken into account when designing a potential target satellite and such design principles will influence future spacecraft designs. As present spacecraft become more and more reliable, their lifespan is often primarily determined by the amount of propellant, used for orbit correction and attitude control maneuvers. In general, to prevent an uncontrollable spacecraft on potential valuable orbits, the remaining propellant is used for a final orbit maneuver to lift the satellite onto a so-called graveyard orbit to free the valuable orbit position. At this time, the satellite's systems are still functioning and would continue to do for years; nevertheless, the GEO box (a range of $+-0.1^{\circ}$ longitude) must not be blocked for other satellites. The increasing number of debris objects in all orbit heights, reduce a satellite's lifetime even more, as more propellant-consuming evasive orbit maneuvers become necessary. In-space refueling would enable space assets to extend their mission until malfunction of vital subsystems or official mission termination. In general, lifetime-extension is possible through human spaceflight, and it has already been successfully demonstrated by NASA with the repair and upgrade of HST). However, the associated costs often render it economically inefficient for less valuable spacecraft. If OOS should turn into a business case, it must be implemented at a price level that makes it interesting for space asset owners to contract an OOS provider for lifetime extension instead of procuring a new satellite. Thus, it needs to be done with small non-expensive service satellites. Furthermore, techniques as autonomous relative navigation in far and close distances as well as machine vision-based automatic docking to even non-cooperative targets will have to be applied. A high degree of autonomy is targeted to reduce monitoring and commanding activities, and thus to saves mission cost.

The two DLR institutes German Space Operation Center (GSOC) and the Institute for Robotics and Mechatronics are currently assigned for ground operations of two missions for in-space demonstration of OOS-keytechnologies. The main objectives of the German DEutsche Orbitale Servicing Mission (DEOS), currently planed to launch in 2013, are:

1. Controlled capturing of a tumbling non-cooperative satellite (Client) by a servicing satellite (Servicer) with an integrated manipulator

2. Disposal of the mated satellite configuration by a controlled reentry maneuver where both satellites are completely burning up

Two experimental satellites will be used to exercise GPS- and machine vision-based autonomous relative navigation methods to rendezvous with a target from various initial conditions in LEO. Robotic telepresence operations will be performed for flexible and rigid coupling of the Servicer to the Target with a ground-controlled robotic manipulator fixed to the servicing satellite. The mission will be completed by a controlled reentry of the coupled satellites. 
The mission Orbit Life Extension Vehicle (OLEV) under lead by a European industrial consortium with a strong DLR participation aims at providing technologies and services to enable the business case of lifetime extension of geostationary communication satellites. Motivation for this is that nowadays communication satellites need to be lifted onto a graveyard orbit when their propellant supply is almost depleted and thus necessary station keeping maneuvers cannot be done any more, albeit they have enough electrical power and transponder capacity for further years of operation. Precise station keeping is vital for OLEV because, depending on the geographical longitude, the geostationary belt around Earth is densely populated and often the space in one box is shared between multiple satellites. Orbit positions in GEO are valuable and communication satellites are expensive, and that's why satellites in danger of becoming uncontrollable need to be placed onto a safe graveyard orbit so that they cannot harm other satellites by drifting uncontrollably. The owner of a communication satellite, of course, also has an economical interest in keeping it in operation as long as possible because every additional month of service lowers the total operation cost and thus increases profit. To facilitate these requirements, the OLEV mission is designed as follows:

1. Piggy-back launch into Geostationary Transfer Orbit (GTO, frequent cost-efficient launch opportunities)

2. Fuel-efficient transfer from GTO to target satellite in GEO by electric propulsion

3. Autonomous machine-vision-based docking on the target satellite's apogee motor nozzle

4. Orbit lifetime extension through taking over station keeping and attitude control tasks

5. Transfer of the target satellite onto a graveyard orbit

6. Undocking and docking onto another GTO-satellite to extend its lifetime (if enough propellant left)

This work focuses on the flight operations specific aspects of both missions. The following section therefore briefly shows the general and mission-specific requirements to GSOC, followed by a description of the ground system architecture, and the planned flight operation organization in the final section. A more detailed description of both missions as well as of the involved technologies and the necessary test and simulation can be found in Ref. 2.

\section{Requirements to GSOC}

GSOC, and its ground station in Weilheim, has experience for decades from a variety of scientific and commercial satellite missions in LEO and GEO. DEOS and OLEV, however, pose challenging new requirements to the ground system infrastructure as well as to the flight operations organization. In this section, first the general requirements common to both missions are treated, followed by the mission-specific requirements of DEOS and OLEV.

\section{A. General Requirements}

Key to success for both missions is the effective implementation of in-space telepresence. This concept aims at enabling a remote operator to control a robotic manipulation element installed on a space asset, as if it were on ground in direct proximity of the operator. Both missions additionally make use of sophisticated technologies like autonomous formation flying and rendezvous, proximity operations of two spacecraft, the capture of or docking to another satellite, called target satellite or just target. This target may be significantly larger than the servicer (OLEV) and/or less cooperative (DEOS), or even both. Because of the necessary computation power for the image processing, the control commands of the robotic manipulators in space are generated on ground, based on video data obtained from the cameras aboard the respective service satellite. In critical mission phases it is also advantageous for the control team to be able to visually supervise the proximity operations in real time. Planed interaction from ground as well as the ability to abort any autonomous actions in case of a flight safety regulation violation is also supported by real time video information. The video transmission from space to ground and the simultaneous transmission of spacecraft and manipulator (DEOS) commands result in the requirement of significantly higher bandwidths than is necessary for regular command operations. The development, the supervision and control of the robotic manipulation elements in space during mission execution phase is done by the Institute of Robotics and Mechatronics. The respective control personnel are to be integrated into the Flight Operations Team (FOT) and definite regulations for the hand over of command authority need to be developed. The general requirements to GSOC for both missions are therefore:

1. Develop and integrate a ground system infrastructure to enable bi-directional high bandwidth communication with latencies of less than $500 \mathrm{~ms}$ (telepresence)

2. Integration of an externally developed Payload Control Center into the Flight Operations Infrastructure 


\section{B. DEOS specific requirements}

For DEOS, two satellites, flying in formation or in coupled configuration, must be monitored and operated. GSOC is required to support independent but also coordinated control of both spacecraft throughout the whole mission lifetime.

Due to orbit heights at LEO altitudes, the effective contact duration is between three and 12 minutes, which render precise ground station network scheduling and experiment planning essential for mission success. The real time video transmission in combination with high data rate control commands of the robotic manipulator and the latency requirement of less than $500 \mathrm{~ms}$ are the main challenge for GSOC. Although overlapping interruption-free contacts are not required, at least two additional antennas contacts are necessary, one or two before and one after the main experiment contact. The preceding contacts are to assure that both spacecraft are in their predefined initial condition. In case of unexpected situations, the flight control can initiate an abort manually. The experiment is carried out during the main contact. In the subsequent contact the flight control team checks if the satellites are in a safe configuration, either in autonomous formation flight or in a coupled configuration. Depending on the respective experiment, the telepresence connection needs to be established during the middle contact. The complex experiments at LEO-altitudes in combination with only short contact durations therefore make a precise and flexible mission planning mandatory for a safe and effective experiment.

At the end of mission execution phase, the final experiment of a controlled re-entry of the two satellites in a rigidly coupled configuration is to be supported by GSOC. As the telemetry transmitted during re-entry is of scientific interest, the maneuver needs to be done within reception range of a ground station. At the same time it must be carried out with maximum safety, i.e. not over populated area, in case small parts survive the descent through the atmosphere.

\section{OLEV specific requirements}

The mission goal of OLEV is to demonstrate economic lifetime extension by taking over the task of attitude control and station keeping for another, usually heavier, satellite. A decisive element for mission success is therefore a close coordination with the client satellite control center. GSOC needs to develop robust processes to assure command authority for routine as well as for contingency operations. The positioning and safety requirements are demanding because the potential target satellite might be in a GEO box with other communication satellites which constrains the space for maneuvering when approaching the target. Details on necessary orbit determination accuracy can be found in Ref 2 .

\section{Ground System Infrastructure}

The planned Ground System Infrastructure is briefly presented and can be studied in more detail in Ref 2 . The requirement of telepresence in space leads to the necessity of high-bandwidth connections with short latencies. The existing command and control system at GSOC, SCOS 2000, does not support such high data rates, and thus the high rate telemetry, the real time video stream and also the high rate telecommands for a space-based robotic manipulator, cannot be routed through. Fig. 1 shows the layout of the planned new connection infrastructure and associated data flows. On-board the spacecraft, the different information types are written into a packetized frame structure and sequentially transmitted to ground. The receiving ground station then extracts the data packets and separates them in low and high rate telemetry. To do so, the different data types need to be distinguishable, which is assured by an embedded IP-protocol for video and robotic telemetry. This technology has been successfully validated in space with the ROCKVISS-experiment at the International Space Station (ISS).

GSOC and its Weilheim ground station are connected through a standard 2 Mbps line, also called multi mission line. This is the standard connection for data transmission for GSOC's satellite missions but not capable enough for telepresence. Another faster, $34 \mathrm{Mbps}$ leased line connection is foreseen for high rate telemetry and high rate telecommands. It bypasses the multi mission line and is used in mission phases when high data rates are needed, which are usually the telepresence phases. It is not possible to use both lines simultaneously. That is why in telepresence phases the normal, low-rate telemetry and telecommands need to be transmitted in one data stream, together with the video and the manipulator commands. A merger is being developed to mix the different outgoing data types, and to the extract incoming data stream parts, and to distribute them correctly to the Payload Control System and SCOS 2000. Besides this peculiarity, the requirements to the ground system infrastructure are in general the same as for any other space missions. For the Launch and Early Orbit Phase (LEOP), a dedicated LEOP network of ground stations is needed, which will be more complex than the one used for the commissioning and the experiment phase. The following two subsections show the differences of each mission from a Ground System Infrastructure point of view. 


\section{DEOS Groundsegment Overview MCC/Weilheim}

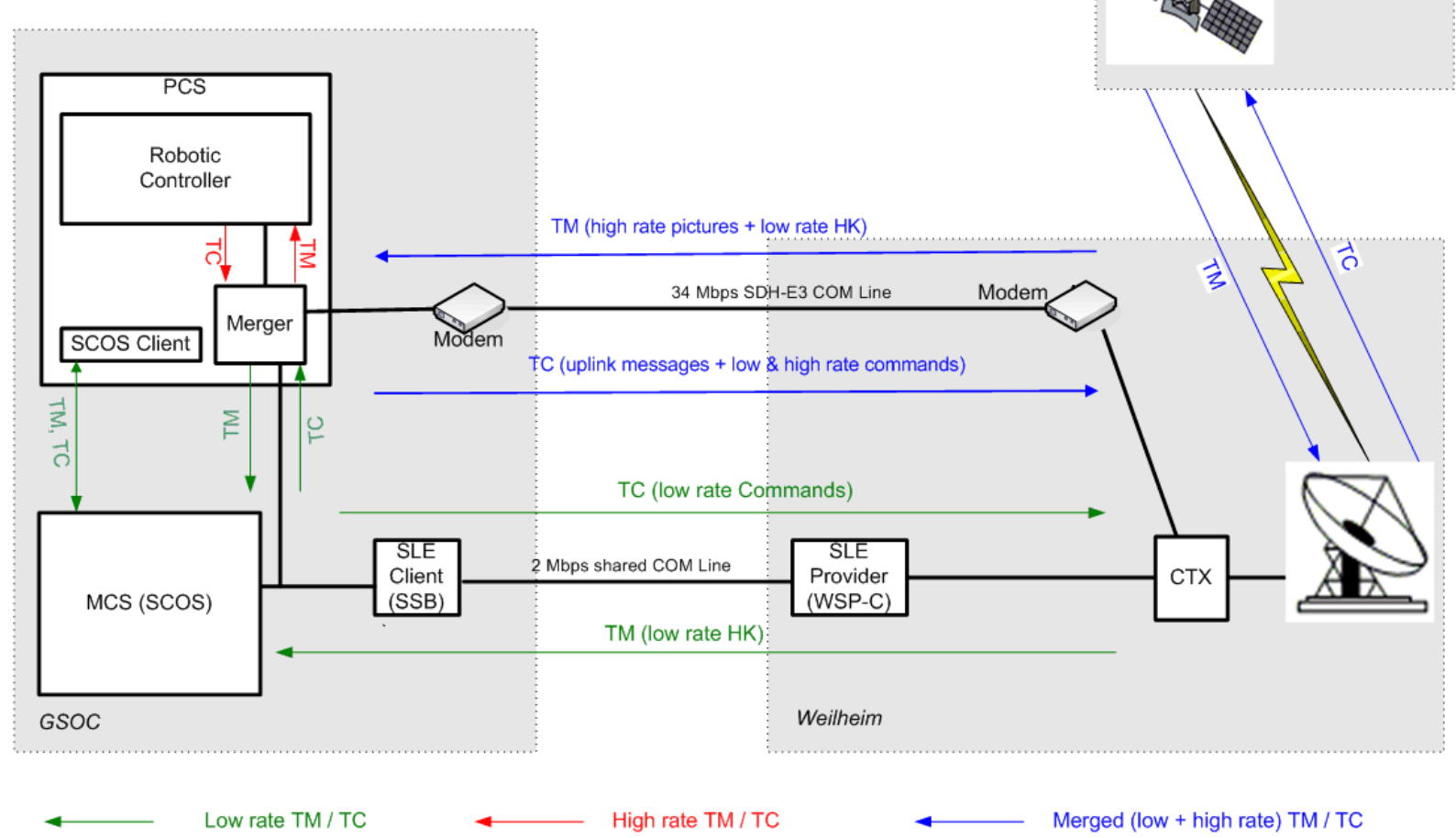

Figure 1: DEOS Ground Segment Infrastructure, which is in general also applicable to OLEV; taken from Ref 2

\section{A. DEOS}

The experiment phase that follows LEOP and Commissioning is planned to last about a year. In this time, the scheduled experiments will be conducted in tests of different duration and intensity. The main ground station Weilheim will be the station with which the actual telepresence experiments will be conducted. Depending on the experiment, one or two additional ground stations are needed before experiment contact, and one more after the Weilheim passage. Candidates for such stations are in Svalbard, Kiruna and in Canada. For some experiments it is advantageous to use a ground station for the first contact that is also capable of receiving high rate telemetry and routing it to GSOC. This enables the flight dynamics and payload operation team to use the video information to assess the spacecraft status before critical experiments are started. Ideally, the passages before and after the main contact should be interruption free, to enable the control team to quickly intervene in critical situations. DEOS requires high data rates not only for video transmission but also for commanding the robotic manipulator. The required bandwidth for this task is about $256 \mathrm{kbps}$ and must be supported by any ground station that will be used for an experiment contact.

\section{B. OLEV}

Compared to DEOS, the ground station requirements are less demanding. The spacecraft will be piggy-back launched and therefore on a GTO after separation. After successful LEOP and commissioning phase, the low-thrust propulsion system is activated. In approximately 160 days the spacecraft then spirals out to its target satellite in GEO. During this phase only a few contacts, at maximum once per day, are necessary to do ranging, to monitor and check the system states, and to command updated steering commands. The final GEO height then allows continuous ground station coverage, which significantly improves mission safety. The target satellite is controlled from another control center. From there the target satellite's attitude control subsystem is deactivated, if the OLEV spacecraft has successfully docked. While approaching the target and during the docking maneuver, close coordination and continuous communication between the two control centers is essential. Normally the target satellite's control center does ranging with the satellites it controls. Depending on the spatial separation between this and the GSOC ground 
station, precise two-way ranging supports the critical mission phase of closing in on the target satellite and docking autonomously to its apogee motor nozzle.

\section{Flight Operations}

\section{A. Operational Concept}

On the bases of the general concept at GSOC for operating unmanned space missions, the mission-specific details of DEOS and OLEV comprise the following.

All control room activities to be managed by a flight director, responsible for flight operations. He/she coordinates all activities comprising the pass preparations, execution of flight procedures during ground station visibilities and the post-pass wrap-up and post processing. He/she is supported by a team of flight operation engineers, organized in the Flight Operations Team (FOT). They monitor all activities of the satellite and its subsystems. They prepare commands for scheduled or unscheduled subsystem-related activities and carry out telemetry data checks during the pass and also, for analysis purposes, after a pass from recorded telemetry data. The only instance from where commands are sent to the spacecraft is the operator position at the special, so-called command console. On order of the flight director, the operator releases a command for transmission to the spacecraft and also monitors its correct reception aboard. During critical mission phases (e.g. LEOP and Commissioning), the FOT is supported by a Satellite Support Team (SST), a team of specialists sent by the spacecraft manufacturer. They assist the FOT with their more comprehensive knowledge of each subsystem and also monitor telemetry data. Top level representatives (e.g. of the satellite owner, the overall project manager, etc.) are also involved, mostly for decision-making in critical situations like major anomalies with a high risk of mission failure.

For the standard mission phases without telepresence activities, the concept described above is sufficient. The spacecraft is monitored and commanded in a proven way via the TM/TC-system SCOS2000, because high data rates are not necessary at this time. For the on-orbit servicing activities, with its higher bandwidth requirement, this concept needs to be enhanced. The Payload Control System PCS will generate control commands for the RvD and robotic activities. These commands bypass the standard command system, which implies to hand over the command authority to the PCS operators. During these phases, any bus-related operation will be paused except the monitoring task. The FOT's responsibility in this mission phase is to assure the readiness to quickly interrupt and resume satellite control in case of violated safety criteria.

\section{OLEV}

The orbit of OLEV (GTO, GEO) allows almost permanent contact with the spacecraft, which, in comparison to DEOS, eases mission operations. The critical telepresence operations start when the transfer to the target satellite is completed and orbit control has changed to relative navigation. From then, the PCS is in the control loop. All activities of this phase need to be coordinated with the client. That's why, during the final approach, GSOC will be in permanent voice contact with the target satellite control center. Telepresence operations will end with the successful docking. From then and until a possible separation, the mated OLEV-Client configuration is operated as a single satellite. Telepresence will be needed again, when undocking from the client is requested and for docking to a new client.

\section{DEOS}

The DEOS experiments will take place in LEO orbit heights, which makes mission operations different to those of OLEV. They are typically characterized by long offline periods and relatively short contact phases. Thus, a time window in the order of only eight minutes is usable for telepresence and robotic activities. Another peculiarity is the simultaneous operation of two satellites that are either in formation flight or telepresence-supported phases of robotic activity. The approach is therefore to use two FOTs, a larger one to operate the Servicer and a smaller one for the Client. The team staffing is presented in more detail in section C.

Figure 2 shows the roles for the control room activities and the flow of orders and telecommands during standard and telepresence activities. Standard TM is available for all positions in the control room during all mission phases. During telepresence periods video and robotic related data are routed to and RvD and robotic TCs are sent from the PCS directly via the telepresence connection. Not respected in the figure are additional positions for simultaneous operations of the target satellite (DEOS). 


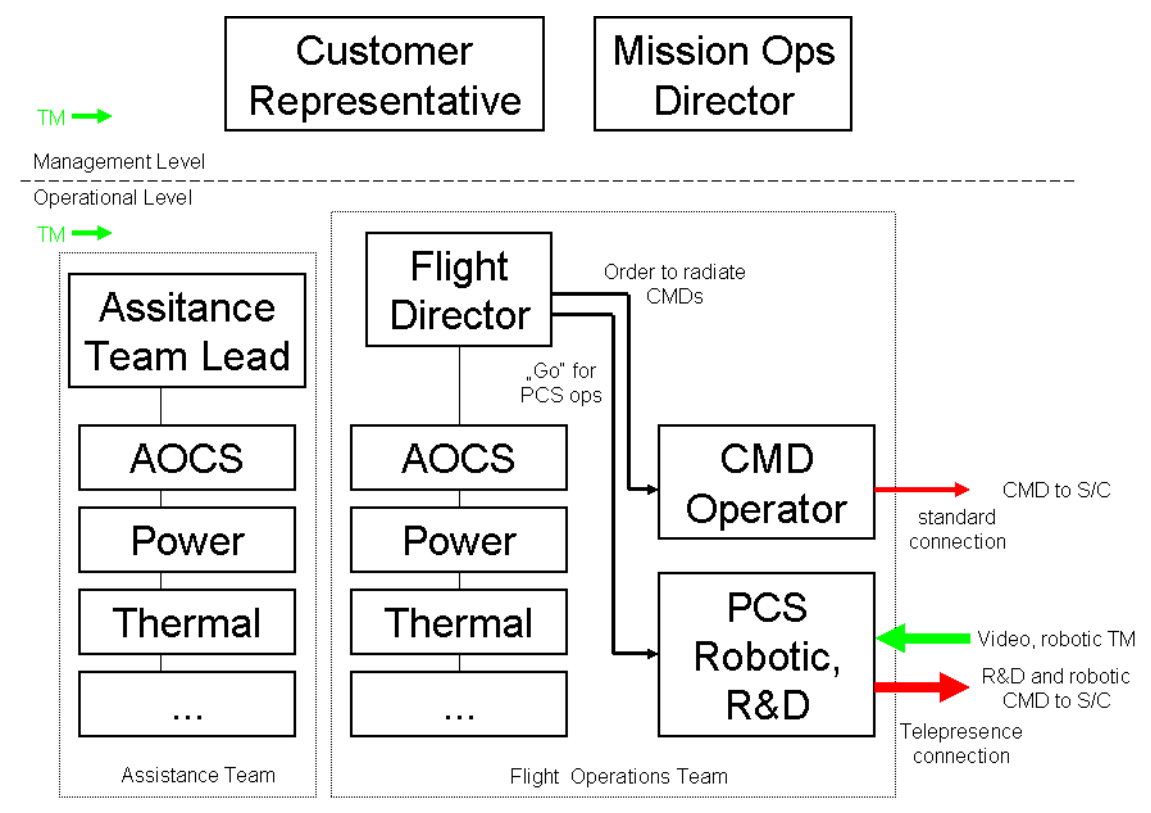

Figure 2: Control room roles and command flow

\section{B. Basic Mission Phases}

OLEV and DEOS have common mission phases, or phases with similar activities, but also exhibit significant differences in mission operations. Each phase is presented and the similarities as well as the mission-specific aspects are explained.

\section{LEOP \& Commissioning}

Both missions will begin with short LEOP phase in which the first contact is established and the propulsion system and other basic functions are initiated. For both missions, early testing of the telepresence capability is an important topic to confirm proper reception of video data and fast commanding.

The commissioning of the OLEV spacecraft comprises the test and calibration of the electric propulsion system, which will be used later in transfer phase. The expected duration for LEOP and commissioning is approximately 3 weeks.

The activities for DEOS are more complex. During launch, Servicer and Client are in a so-called mated configuration. The early tests and calibrations are done with the Servicer first whilst the Client remains passive. After separation from the Servicer, the Client is then operated and tested independently. It is foreseen to separate both spacecrafts in telepresence mode. This way, a certain spatial distance can be attained in a controlled manner. Finally, a passively safe formation of both satellites should be assured.

Before starting any further activity, the test of Collision Avoidance Maneuvers (CAM) is mandatory for both missions. In case of critical situations during RvD (Rendezvous and Docking) or docking, such commands would be later initiated by ground or autonomously by the spacecraft to prevent damage to the satellites.

\section{Transfer}

An electrical propulsion transfer phase of about 180 days is planned for OLEV to bring the spacecraft from GTO close to the client satellite in GEO. Once the low thrust activities have started, the team size can be reduced.

\section{RvD, Robotic Ops}

For both missions, this is the critical and most challenging phase. A rendezvous of two satellites is done, followed by docking or close proximity operations. This requires adequate strategies and mechanisms to avoid a collision which are discussed in section D.

The RvD operations for OLEV start at a distance of one kilometer with from the client. The telepresence operations start and the distance between both satellites is reduced using relative navigation. For the final approach, GSOC stays in contact with the customer satellite control center. Shortly before docking, the target satellite will freeze its AOCS control in order prevent unexpected and, at this time, unwanted reactions. After successful docking, 
telepresence operations are terminated and further operations are carried out with regular space operations. The dynamic behavior of the now mated configuration is tested and the Servicer's AOCS subsystem calibrated. This phase is planned to last about 10 days.

Telepresence operations are resumed again when an undocking and the separation from the client satellite is planned or when the next customer is approached.

The robotic operations with DEOS will start right after completion of the commission phase. Several experiments are planned to exercise rendezvous and robotic activities. Telepresence activities, which can be only be done via GSOC's Weilheim station, need to be commenced by preparation passes with other ground stations 1030 min beforehand and finalized by a consecutive pass for confirmation of a save post test configuration . The activities and their timing are:

- Preparation passes

1-2 passes shortly before the telepresence pass

Check of proper approach to the Client; corrections or abort commands are sent to the Servicer; the Client is commanded into desired attitude (e.g. without rotations, single axis rotating, tumbling, etc.);

Passes are performed in standard mode.

- Telepresence activity

Single pass used for the robotic activities

Pass is performed in telepresence mode

- Confirmation pass

Single pass within 30 min after telepresence pass for final check of safe constellation

Pass is done in standard mode again.

\section{Routine}

A routine operations phase is only foreseen for OLEV. The phase starts after successful docking to the client satellite and successful checkout of the AOCS functions in mated configuration. The customer spacecraft is kept within the GEO box as long as required which might be some years. Since the client spacecraft is usually not alone in the box, a close cooperation with the client control center is required and station keeping maneuvers need to be coordinated accordingly.

A change to a different location in GEO on request is also possible.

\section{Disposal}

For the OLEV mission, this phase means disposal of the client satellite. It starts, when the customer decides to terminate the client satellite's operation. The necessary transfer onto a graveyard orbit will be done with OLEV's propulsion system. If there is enough fuel left for extending another satellite's lifetime, OLEV will undock and return to GEO for the next customer. At the end of OLEV's lifetime, OLEV will stay in graveyard orbit together with the last client.

As a final experiment, DEOS will complete its mission by a controlled reentry. After a last capture of the Client, both satellites will stay in coupled configuration. The hydrazine system of the Client is then used for the required orbit corrections to attain an orbit that leads to reentry into the Earth's atmosphere.

\section{Staffing}

The basic staffing approach for both missions is that all activities would be covered by one shift per day. Thus, only one flight operations team needs to be built up, trained, and maintained. Outside of this so called main shift, and during no activity periods (e.g. at nights or at weekends), command operators will monitor the satellites and do routine or uncritical commanding whilst key personnel is on call in case of anomalies.

Due to the early states of both projects, exact numbers for required personnel, especially for the subsystem specialists, cannot be given yet. The spacecraft design, neither for OLEV nor DEOS, is not finalized yet and the effort to operate them can only be estimated, but at least a qualitative assessment can be done.

\section{LEOP Team}

During LEOP and the first days of commissioning, the full team, consisting of personnel as described in section A, will be present. It is expected to have additional specialists from the S/C manufacturer and more top level representatives available. Therefore, the flight director is supervised and supported by the project's mission operations director who is in close contact with the overall project management.

For the separation of DEOS Servicer and Client, simultaneous operation of two satellites is required. Therefore, two operations teams need to be built, one larger team operating the Servicer and doing the telepresence activities, 
and one small team responsible for the client spacecraft. The small team will consist of one command operator and at least one responsible system or subsystem engineer.

\section{Telepresence Team}

For telepresence operations, the full operations team, as described for LEOP will be present. The team will be complemented by additional personnel responsible for the docking/robotic hard- and software at the PCS console.

\section{Routine Team}

In general, the routine team will typically cover phases of little activities. Monitoring and routine commanding of the spacecraft(s) are performed by the command operators. They will work on shift to provide a 24/7 coverage. Anomalies are reported to the flight director. He and the subsystem specialists will be at GSOC during normal working hours doing offline analysis and planning of upcoming events. An on-call plan will be active for unexpected occurrences.

During routine activities, e.g. orbit maneuvers or maintenance activities during routine phase or during transfer phase, additional specialists (e.g. AOCS) will be present if required. For DEOS, also the autonomous formation flight between two consecutive test campaigns needs to be monitored by the routine team.

\section{Mission Safety Aspects}

The most critical aspect of both missions is to fly two satellites in close formation. The unplanned contact of both spacecrafts must be avoided by all means. Key functions to avoid collisions will be:

- A reliable close formation flying mode (for DEOS) where both spacecraft can be kept in a passively safe formation for a longer time with a minimized risk of unintended contact between the spacecraft

- A collision avoidance mode or maneuver (CAM) that will bring the chasing S/C (OLEV or DEOS Servicer) in a safe distance to the target. Reasons for such situations could be a disruption of the telepresence connection, problems of ground software during final approach or others. A CAM could be initiated by ground command or autonomously onboard via FDIR mechanisms.

Another new aspect of this kind of missions is operation of the spacecraft with ground control in-the-loop. The PCS console receives video and RvD related telemetry data and generates direct control commands for AOCS and the robotic arm (DEOS only). During telepresence, the control of the S/C is fully in the hands of the PCS engineers. The remaining flight ops team has of course still the ability to sent standard satellite bus related commands if necessary and can initiate a CAM. Smooth interaction between the teams is mandatory and needs to be trained intensively.

The LEO characteristic of the DEOS mission shall be mentioned here. The DEOS Servicer needs much more autonomy than OLEV. Most of the time, it will operate without ground contact and will do the final approach to the Client autonomously. The ground team can only do intermediate checks before the actual telepresence pass.

\section{Conclusion}

Both missions, OLEV and DEOS, are challenging technically and also with respect to the flight operations. Therefore, new concepts needed to be developed at GSOC to enable successful mission operation.

Due to the closer distance to the ground station and consequently shorter delay times between satellite and ground control, robotic telepresence activities can be better realized in LEO than in GEO. On the other hand, the short contact phases in LEO are critical and require a highly developed autonomy of the servicing spacecraft and a well prepared and trained ground control team. In either case, unintended contacts between the involved spacecraft should be avoided. For DEOS, damaged satellites might prevent further experimental activities, a collision with OLEV will probably fray the client beyond repair. Also, generation of additional debris in GEO must be avoided. A safe handling of the satellites will be the key element for successful accomplishment. An in-depth preparation of the ground segment, in combination with an extensive simulation of telepresence activities, and the validation of abort and collision avoidance mechanism and routines, is therefore mandatory. The current status of these developments at GSOC has been presented.

After discussing the flight operations approach for two ambitious missions, a short status of both projects is given here. The OLEV concept development resulted in a feasibility study and a critical design based on the SMART satellite bus of the Swedish Space Cooperation SSC. For DEOS project phases 0 and A were successfully performed. The preliminary design phase started in 2010.

GSOC is prepared to operate both on-orbit servicing missions. 


\section{Acknowledgments}

The DEOS mission phase-A study has been awarded by the Space Agency of the Aerospace Center (DLR) and funded by the German Federal Ministry for Economics and Technology (Förderkennzeichen 50 RA 0802).

\section{References}

${ }^{1}$ Settelmeyer, E., Lehrl, E., Oesterlein, W., Hartmann, R., Landzettel, K., „The Experimental Servicing Satellite - ESS“, IARP 98-c-14, 1998

${ }^{2}$ Sellmaier, F., Boge, T., Spurmann, J., Gully, S., Rupp, T., Huber, F., „On-Orbit Servicing Missions: Challenges and solutions for Spacecraft Operations“, AIAA SpaceOps 2010 Conference, Huntsville, Alabama, USA, 2010-2159 (submitted)

${ }^{3}$ Fehse, W, „Automated Rendezvous and Docking of Spacecraft“, Cambridge Aerospace Series 16, Cambridge University Press, 2003

${ }^{4}$ Fehse, W, „Rendezvous and Docking Workshop“, Oberpfaffenhofen, Germany, 18-19 March 2009 